Acta Crystallographica Section D

Biological

Crystallography

ISSN 1399-0047

Shipeng Wang,‡ Misaki Ogata,‡ Shoichiro Horita, $\neq$ Jun Ohtsuka, Koji Nagata and Masaru Tanokura*

Department of Applied Biological Chemistry, Graduate School of Agricultural and Life Sciences, University of Tokyo, 1-1-1 Yayoi, Bunkyo-ku, Tokyo 113-8657, Japan

\# The first three authors made equal contributions.

Correspondence e-mail: amtanok@mail.ecc.u-tokyo.ac.jp

\title{
A novel mode of ferric ion coordination by the periplasmic ferric ion-binding subunit FbpA of an ABC-type iron transporter from Thermus thermophilus $\mathrm{HB8}$
}

Crystal structures of FbpA, the periplasmic ferric ion-binding protein of an iron-uptake $\mathrm{ABC}$ transporter, from Thermus thermophilus HB8 (TtFbpA) have been solved in apo and ferric ion-bound forms at 1.8 and $1.7 \AA$ resolution, respectively. The latter crystal structure shows that the bound ferric ion forms a novel six-coordinated complex with three tyrosine side chains, two bicarbonates and a water molecule in the metal-binding site. The results of gel-filtration chromatography and dynamic light scattering show that TtFbpA exists as a monomer in solution regardless of ferric ion binding and that TtFbpA adopts a more compact conformation in the ferric ion-bound state than in the apo state in solution.

\section{Introduction}

Iron is an essential element for the growth and survival of nearly all living organisms. However, it is difficult for most organisms to obtain sufficient iron from the environment because of the extremely low solubility of ferric ion (Braun \& Killmann, 1999). One of the strategies for iron acquisition is to use the ATP-binding cassette (ABC) transport system. In Gram-negative bacteria, a typical iron-uptake $\mathrm{ABC}$ transporter consists of a ferric ion-binding protein (Fbp) located in the periplasm (FbpA), two transmembrane proteins that form a pathway for ferric ions ( $\mathrm{FbpB})$ and two peripheral ATPbinding proteins located on the cytoplasmic side of the inner membrane (FbpC) (Hollenstein et al., 2007; Gerber et al., 2008).

Based on the crystal structures of ferric ion-binding proteins from Gram-negative bacteria, four types of coordination to ferric ion have been reported thus far: (i) HiFbpA from Haemophilus influenzae utilizes two tyrosine residues, a glutamate residue and a histidine residue (class 1; Bruns et al., 1997, 2001); (ii) YfuA from Yersinia enterocolitica utilizes two tyrosine residues, an aspartate residue and a histidine residue (class 2; Shouldice et al., 2005); (iii) MhFbpA from Mannheimia haemolytica (formerly Pasteurella haemolytica) utilizes three tyrosine residues (class 3; Shouldice et al., 2003, 2004); and (iv) cFbpA from Campylobacter jejuni utilizes four tyrosine residues and a histidine residue (class 4; Tom-Yew et al., 2005).

TTHA1628-TTHA1629-TTHA1630 is a putative ironuptake ABC transporter from Thermus thermophilus HB8 (Kim et al., 2012). TTHA1628 (TtFbpA), the ferric ionbinding protein consisting of 311 amino-acid residues, shows a specific affinity for ferric ion(s) (Wang et al., 2011). Here, we report the crystal structures of the apo form and ferric ion-bound form of TtFbpA at 1.8 and $1.7 \AA$ resolution, respectively. The crystal structure of the ferric ion-bound TtFbpA shows that a ferric ion binds to a specific site of
Received 17 May 2013

Accepted 23 September 2013

PDB references: TtFbpA, apo, 3wae; ferric ion-bound, 3waf 
Table 1

Crystallographic data-collection and refinement statistics for apo and ferric ion-bound TtFbpA.

Values in parentheses are for the highest resolution shell.

\begin{tabular}{llll}
\hline & Apo TtFbpA & $\mathrm{Fe}^{3+}$-bicarbonates-TtFbpA \\
\hline X-ray data collection & & & \\
Source & NW12A, PF-AR & BL32XU, SPring-8 & NE3A, PF-AR \\
Wavelength $(\AA)$ & 1.0000 & 1.0000 & 1.7209 \\
Resolution $(\AA)$ & $20-1.80(1.85-1.80)$ & $45-1.69(1.79-1.69)$ & $20-2.60(2.67-2.60)$ \\
Space group & $P 2_{1} 2_{1} 2_{1}$ & $P 2_{1} 2_{1} 2_{1}$ & $P 2_{1} 2_{1} 2_{1}$ \\
Unit-cell parameters $(\AA)$ & $a=39.15, b=99.05$, & $a=40.17, b=101.0$, & $a=39.00, b=99.04$, \\
& $c=132.2$ & $c=134.6$ & $c=132.5$ \\
$V_{\mathrm{M}}\left(\AA^{3} \mathrm{Da}^{-1}\right)$ & 1.88 & 2.00 & 1.87 \\
Unique reflections & 47714 & 62381 & 30213 \\
Multiplicity & $13.2(10.2)$ & $7.0(6.3)$ & $3.7(3.5)$ \\
Completeness $(\%)$ & $98.5(89.6)$ & $99.4(96.4)$ & $99.2(96.2)$ \\
$R_{\text {merge }}(\%)$ & $5.6(46.5)$ & $7.9(57.7)$ & $9.3(46.3)$ \\
$\langle I / \sigma(I)\rangle$ & $30.8(5.0)$ & $17.3(2.9)$ & $12.3(2.5)$ \\
Refinement & & & \\
PDB code & 3 wae & 3 waf & \\
Resolution $(\AA)$ & $20-1.80$ & $45-1.70$ & \\
$R_{\text {work }} / R_{\text {free }}(\%)$ & $17.1 / 20.5$ & $20.0 / 24.1$ & \\
R.m.s.d., bond lengths $(\AA)$ & 0.011 & 0.009 & \\
R.m.s.d., bond angles $\left({ }^{\circ}\right)$ & 1.426 & 1.306 & \\
No. of non-H atoms & & & \\
Fe & 0 & 2 & \\
Bicarbonate & 0 & 16 & \\
Protein & 4792 & 4821 & \\
2-Propanol & 16 & 513 & \\
Water & 292 & 94 & \\
Ramachandran plot, residues in $(\%)$ & 93 & & \\
Most favoured regions & 7 & & \\
Additionally favoured regions & 7 & & \\
\hline
\end{tabular}

$\dagger R_{\text {merge }}=\sum_{h k l} \sum_{i}\left|I_{i}(h k l)-\langle I(h k l)\rangle\right| / \sum_{h k l} \sum_{i} I_{i}(h k l)$, where $I_{i}(h k l)$ is the $i$ th observation of reflection $h k l$ and $\langle I(h k l)\rangle$ is the weighted average intensity for all observations $i$ of reflection $h k l$. $\ddagger$ The $R_{\text {free }}$ factor was calculated using $5 \%$ of reflections omitted from the refinement.

TtFbpA to form a six-coordinated complex with three tyrosine residues, two bicarbonates and a water molecule, revealing a novel mode of coordination to a ferric ion. Very recently, crystal structures of TtFbpA in three apo forms and one ferric ion-bound form have been reported (Wang et al., 2013). However, these TtFbpA structures are different from our TtFbpA structures not only in the unit-cell parameters and the space group but also in the manner of ferric ion coordination. In these structures the ferric ion was five-coordinated in a manner similar to the binding of ferric ion by MhFbpA. The reasons for these different types of coordination are discussed.

\section{Materials and methods}

\subsection{Cloning, expression and purification}

The gene encoding FbpA from T. thermophilus HB8 (TtFbpA) was cloned into pRSFDuet-1 (Novagen) using an In-Fusion PCR Cloning System (Clontech). The PCR primers were designed to remove the putative signal sequence at the $\mathrm{N}$-terminus (amino-acid residues 1-19). The DNA sequence of the mature TtFbpA-encoding region of the resulting plasmid was verified. TtFbpA without any tag was overexpressed in Escherichia coli Rosetta (DE3) cells (Novagen) grown in 21 LB medium at $310 \mathrm{~K}$. Protein expression was induced by adding isopropyl $\beta$-D-1-thiogalactopyranoside (IPTG) to a final concentration of $0.1 \mathrm{~m} M$ at an $\mathrm{OD}_{600}$ of $\sim 0.6$ and the culture was continued for a further $24 \mathrm{~h}$ at $298 \mathrm{~K}$. The harvested cells were resuspended in $100 \mathrm{~m} M$ Tris- $\mathrm{HCl} \mathrm{pH}$ $8.0,150 \mathrm{mM} \mathrm{NaCl}$ with a protease-inhibitor cocktail and disrupted by sonication on ice. The lysate was centrifuged at $40000 \mathrm{~g}$ at $277 \mathrm{~K}$ for $60 \mathrm{~min}$ to obtain the soluble fraction. The following procedures were performed at room temperature ( $\sim 298 \mathrm{~K})$ unless otherwise stated. The soluble fraction was incubated at $363 \mathrm{~K}$ for $30 \mathrm{~min}$ and centrifuged at $40000 \mathrm{~g}$ at $277 \mathrm{~K}$ for $60 \mathrm{~min}$ to remove aggregated heat-labile proteins. A final concentration of $0.3 \%(v / v)$ polyethylenimine was added to the supernatant to precipitate nucleic acids and the suspension was centrifuged at $40000 \mathrm{~g}$ at $277 \mathrm{~K}$ for $60 \mathrm{~min}$ to remove the precipitate. A 1.6-fold volume of saturated ammonium sulfate solution was then added to the TtFbpA solution, resulting in a $27 \%$ saturation of ammonium sulfate. The mixture was stirred for $30 \mathrm{~min}$ at $277 \mathrm{~K}$ and centrifuged at $40000 \mathrm{~g}$ at $277 \mathrm{~K}$ for $90 \mathrm{~min}$. The precipitate was dissolved in $100 \mathrm{~m} M$ Tris- $\mathrm{HCl}$ pH 8.0, $150 \mathrm{~m} M$ $\mathrm{NaCl}$. The solution was loaded onto a Superdex 200 10/30 HR (GE Healthcare) column pre-equilibrated with the same buffer at a flow rate of $0.4 \mathrm{ml} \mathrm{min}^{-1}$. To obtain ferric ion-bound TtFbpA samples, ninefold molar excesses of ferric chloride hexahydrate $\left(\mathrm{FeCl}_{3} \cdot 6 \mathrm{H}_{2} \mathrm{O}\right)$ and sodium bicarbonate $\left(\mathrm{NaHCO}_{3}\right)$ were added after gel filtration. The purified sample was concentrated to approximately $12 \mathrm{mg} \mathrm{ml}^{-1}$ using a $20 \mathrm{ml}$ Vivaspin concentrator (10 kDa cutoff; Sartorius). The protein concentration was determined based on the absorbance at $280 \mathrm{~nm}$ obtained using a NanoDrop ND-1000 spectrophotometer (Thermo Scientific) and a calculated molar extinction coefficient $\left(\varepsilon_{280}=41370 \mathrm{M}^{-1} \mathrm{~cm}^{-1}\right)$.

\subsection{Crystallization and $\mathrm{X}$-ray diffraction data collection}

Initial crystallization screening was performed in 96-well Intelli-Plates (Art Robbins) using commercially available kits, namely Crystal Screen HT, Index HT (Hampton Research) and Wizard I and II (Emerald BioSystems) at 293 K. A crystallization drop was prepared by mixing $0.75 \mu \mathrm{l}$ protein solution and $0.75 \mu \mathrm{l}$ reservoir solution and was equilibrated against $50 \mu \mathrm{l}$ reservoir solution. After two-dimensional grid optimization of crystallization conditions ( $\mathrm{pH}$ versus precipitant concentration), the crystallization conditions were further optimized by the sitting-drop vapour-diffusion method in 24well Cryschem Plates (Hampton Research). A crystallization drop was prepared by mixing $1.0 \mu \mathrm{l}$ protein solution and $1.0 \mu \mathrm{l}$ 
reservoir solution and was equilibrated against $400 \mu \mathrm{l}$ reservoir solution.

Crystals of apo TtFbpA were obtained using a reservoir composition of $0.1 \mathrm{M}$ sodium citrate $\mathrm{pH}$ 5.2, $20 \%(v / v)$ 2-propanol, 20\%(w/v) PEG 4000. Each crystal was picked up in a mounting loop and cooled in a cold nitrogen-gas stream using a cryoprotectant consisting of $0.1 \mathrm{M}$ sodium citrate $\mathrm{pH}$ 5.2, 20\%(v/v) 2-propanol, $20 \%(w / v)$ PEG 4000, 15\%(v/v) ethylene glycol. X-ray diffraction experiments for apo TtFbpA crystals were performed on beamline NW12A at the Photon Factory-Advanced Ring (PF-AR), Ibaraki, Japan. A data set was collected from an apo TtFbpA crystal with a wavelength of $1.0000 \AA$, a crystal-todetector distance of $166.2 \mathrm{~mm}$, an oscillation angle of $0.5^{\circ}$ and an exposure time of $1 \mathrm{~s}$ per image using an ADSC Quantum 210 detector.

Crystals of ferric ion-bound TtFbpA were obtained with a reservoir composition of $0.1 \mathrm{M}$ bis-tris $\mathrm{pH}$ 5.5, $0.2 \mathrm{M}$ sodium chloride, 25\%(w/v) PEG 3350. Each crystal was picked up in a mounting loop and cooled in a cold nitrogen-gas stream using a cryoprotectant consisting of $0.1 M$ bis-tris $\mathrm{pH}$ 5.5, $0.2 M$ sodium chloride, $25 \%(w / v)$ PEG 3350, 15\%(v/v) ethylene glycol. $\mathrm{X}$-ray diffraction experiments for ferric ion-bound TtFbpA crystals were performed on beamlines BL32XU at SPring-8, Hyogo, Japan and NE3A at
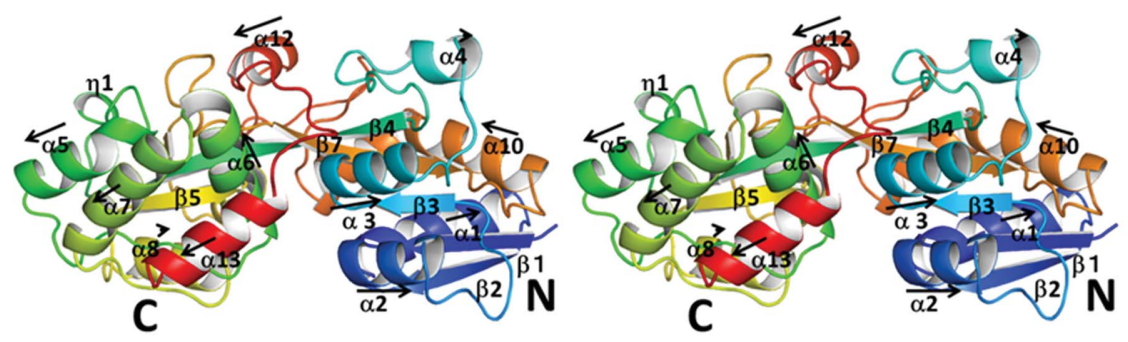

(a)

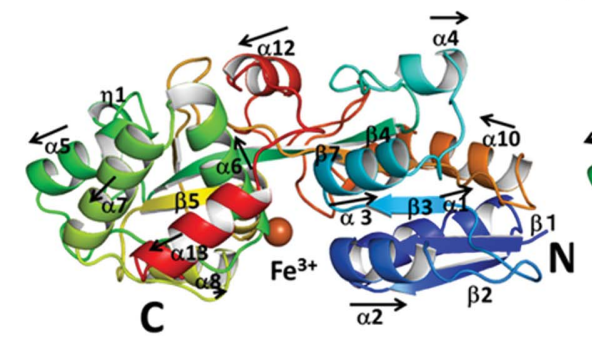

(b)

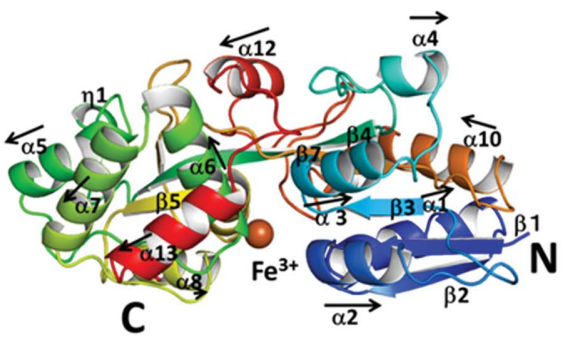

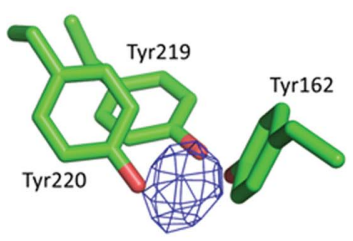

(c)

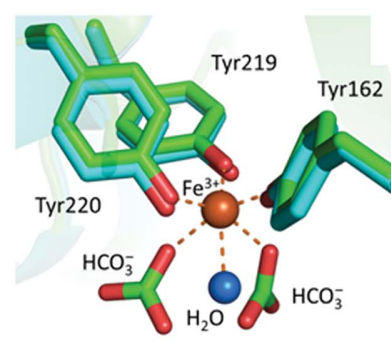

(d)

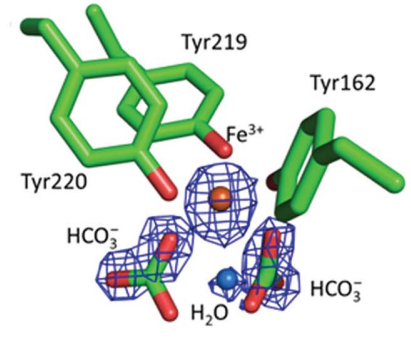

(e)
Figure 1

Crystal structures of TtFbpA. Stereoview of the overall structures of $(a)$ apo TtFbpA and $(b) \mathrm{Fe}^{3+}-$ bicarbonates-TtFbpA. The molecule is shown in rainbow colours from the $\mathrm{N}$-terminus (blue) to the $\mathrm{C}$-terminus (red). $\alpha, \beta$ and $\eta$ represent $\alpha$-helices, $\beta$-strands and $3_{10}$-helices, respectively. (c) Anomalous difference Fourier map of the ferric ion shown in blue mesh contoured at $4.0 \sigma .(d)$ The electron-density OMIT map $\left(2 F_{\mathrm{o}}-F_{\mathrm{c}}\right.$ map shown in blue mesh contoured at $\left.1.5 \sigma\right)$ was superimposed onto the ferric ion-binding site of $\mathrm{Fe}^{3+}$-bicarbonates-TtFbpA. The OMIT map was generated by omitting the atoms of two bicarbonates and a water. (e) Comparison of the ferric ionbinding site of apo TtFbpA (cyan) and $\mathrm{Fe}^{3+}$-bicarbonates-TtFbpA (green). Three tyrosine residues and the bicarbonates are shown as stick models and the ferric ion and a water molecule are shown as spheres.

PF-AR. Data sets were collected from the ferric ion-bound TtFbpA crystals for structure determination and to obtain an anomalous difference Fourier map for ferric ion at wavelengths of 1.0000 and $1.7209 \AA$ with crystal-to-detector distances of 130.0 and $159.4 \mathrm{~mm}$, oscillation angles of 1 and $1^{\circ}$ and exposure times of 1 and $2 \mathrm{~s}$ per image using MAR225HE and ADSC Quantum 270 detectors, respectively.

\subsection{Structure solution and refinement}

The X-ray diffraction data were indexed, integrated and scaled with the $X D S$ program package (Kabsch, 2010). Initial structural models of apo TtFbpA and ferric ion-bound TtFbpA were obtained by molecular replacement with Phaser (McCoy et al., 2007) using the crystal structures of SfuA (32\% sequence identity to TtFbpA; PDB entry 1xvy; Shouldice et al., 2005) and of apo TtFbpA as search models, respectively. Further model building and refinement were performed with ARP/wARP (Morris et al., 2003), Coot (Emsley \& Cowtan, 2004) and REFMAC (Murshudov et al., 2011). The stereo- chemistry of the structure was checked by PROCHECK (Laskowski et al., 1993). The tertiary structure was visualized with PyMOL (DeLano, 2002).

\subsection{Dynamic light scattering}

Dynamic light scattering (DLS) measurements were performed using a DynaPro-801 MSTC (Protein Solutions). The samples of apo TtFbpA and ferric ion-bound TtFbpA were prepared as described above. All samples were filtered through a $0.22 \mu \mathrm{m}$ filter before analysis.

\section{Results and discussion}

\subsection{Structure determination of the apo form of TtFbpA}

Diffraction-quality crystals of apo TtFbpA were obtained with a reservoir composition of $0.1 M$ sodium citrate $\mathrm{pH} 5.2$, $20 \%(w / v)$ 2-propanol, 20\%(w/v) PEG 4000. The apo TtFbpA crystal belonged to space group $P 2{ }_{1} 2_{1} 2_{1}$, with unit-cell parameters $a=39.15, b=99.05, c=132.2 \AA$ (Table 1). The crystal 
Table 2

Geometry of the ferric ion-binding site in TtFbpA.

\begin{tabular}{|c|c|c|}
\hline & Chain $A$ & Chain $B$ \\
\hline \multicolumn{3}{|l|}{ Bond lengths $(\AA)$} \\
\hline $\mathrm{Fe}-\mathrm{OH}(\mathrm{Tyr} 162)$ & 1.93 & 1.89 \\
\hline $\mathrm{Fe}-\mathrm{OH}(\mathrm{Tyr} 219)$ & 1.91 & 1.96 \\
\hline $\mathrm{Fe}-\mathrm{OH}(\mathrm{Tyr} 220)$ & 1.98 & 2.08 \\
\hline $\mathrm{Fe}-\mathrm{OC}\left(\mathrm{HCO}_{3} 332\right)$ & 2.28 & 2.20 \\
\hline $\mathrm{Fe}-\mathrm{OC}\left(\mathrm{HCO}_{3} 333\right)$ & 2.26 & 2.08 \\
\hline $\mathrm{Fe}-\mathrm{OH}(\mathrm{HOH} 511)$ & 2.41 & 2.58 \\
\hline \multicolumn{3}{|l|}{ Bond angles $\left({ }^{\circ}\right)$} \\
\hline $\mathrm{OH}(\mathrm{Tyr} 162)-\mathrm{Fe}-\mathrm{OH}(\mathrm{Tyr} 219)$ & 99.89 & 101.11 \\
\hline $\mathrm{OH}(\mathrm{Tyr} 162)-\mathrm{Fe}-\mathrm{OH}(\mathrm{Tyr} 220)$ & 97.03 & 95.36 \\
\hline $\mathrm{OH}(\mathrm{Tyr} 162)-\mathrm{Fe}-\mathrm{OC}\left(\mathrm{HCO}_{3} 332\right)$ & 87.57 & 94.51 \\
\hline $\mathrm{OH}(\mathrm{Tyr} 162)-\mathrm{Fe}-\mathrm{OC}\left(\mathrm{HCO}_{3} 333\right)$ & 162.30 & 163.48 \\
\hline $\mathrm{OH}(\mathrm{Tyr} 162)-\mathrm{Fe}-\mathrm{OH}(\mathrm{HOH} 511)$ & 86.99 & 84.03 \\
\hline $\mathrm{OH}(\mathrm{Tyr} 219)-\mathrm{Fe}-\mathrm{OH}(\mathrm{Tyr} 220)$ & 115.95 & 119.38 \\
\hline $\mathrm{OH}(\mathrm{Tyr} 219)-\mathrm{Fe}-\mathrm{OC}\left(\mathrm{HCO}_{3} 332\right)$ & 85.36 & 78.56 \\
\hline $\mathrm{OH}\left(\right.$ Tyr219) $-\mathrm{Fe}-\mathrm{OC}\left(\mathrm{HCO}_{3} 333\right)$ & 92.83 & 92.54 \\
\hline $\mathrm{OH}(\mathrm{Tyr} 219)-\mathrm{Fe}-\mathrm{OH}(\mathrm{HOH} 511)$ & 158.73 & 153.90 \\
\hline $\mathrm{OH}(\mathrm{Tyr} 220)-\mathrm{Fe}-\mathrm{OC}\left(\mathrm{HCO}_{3} 332\right)$ & 156.79 & 157.19 \\
\hline $\mathrm{OH}(\mathrm{Tyr} 220)-\mathrm{Fe}-\mathrm{OC}\left(\mathrm{HCO}_{3} 333\right)$ & 88.28 & 85.82 \\
\hline $\mathrm{OH}(\mathrm{Tyr} 220)-\mathrm{Fe}-\mathrm{OH}(\mathrm{HOH} 511)$ & 82.74 & 85.12 \\
\hline $\mathrm{OC}\left(\mathrm{HCO}_{3} 332\right)-\mathrm{Fe}-\mathrm{OC}\left(\mathrm{HCO}_{3} 333\right)$ & 81.20 & 79.01 \\
\hline $\mathrm{OC}\left(\mathrm{HCO}_{3} 332\right)-\mathrm{Fe}-\mathrm{OH}(\mathrm{HOH} 511)$ & 74.77 & 75.52 \\
\hline $\mathrm{OC}\left(\mathrm{HCO}_{3} 333\right)-\mathrm{Fe}-\mathrm{OH}(\mathrm{HOH} 511)$ & 76.91 & 79.64 \\
\hline
\end{tabular}

structure of apo $\mathrm{TtFbpA}$ was determined at $1.8 \AA$ resolution by molecular replacement using the structure of SfuA (PDB entry 1xvy) as the search model. The final structure of apo TtFbpA contained two monomeric TtFbpA molecules (residues 22-330 for both chains $A$ and $B$ ), 292 water molecules and two 2-propanol molecules in the asymmetric unit. No electron density was observed for the two residues (residues 20-21) at the N-terminus. Data-collection and refinement statistics are summarized in Table 1. The Ramachandran plot of the apo TtFbpA structure shows that 93 and $7 \%$ of the residues are in the most favoured and the additionally favoured regions, respectively. The apo TtFbpA protomer contains seven $\beta$-strands, $13 \alpha$-helices and one $3_{10}$-helix (Fig. $1 a)$. The r.m.s.d. value between the two apo TtFbpA protomers in the asymmetric unit was $0.9 \AA$ for $308 \mathrm{C}^{\alpha}$ pairs.

\subsection{Structure determination of the ferric ion-bound form of TtFbpA}

Diffraction-quality crystals of ferric ion-bound TtFbpA were obtained with a reservoir composition of $0.1 M$ bis-tris pH 5.5, $0.2 M$ sodium chloride, 25\%( $w / v)$ PEG 3350. The ferric ion-bound TtFbpA crystal belonged to space group $P 22_{1} 2_{1} 2_{1}$, with unit-cell parameters $a=40.17, b=101.0, c=134.6 \AA$ (Table 1). The crystal structure of ferric ion-bound TtFbpA was determined at $1.7 \AA$ resolution using the molecularreplacement method with the structure of apo TtFbpA as the search model. The final structure of ferric ion-bound TtFbpA contained two monomeric TtFbpA molecules (residues 22330 for chain $A$ and 23-330 for chain $B$ ), 513 water molecules, two ferric ions and four bicarbonate ions in the asymmetric unit. No electron density was observed for two or three residues (residues 20-21 for chain $A$ and residues 20-22 for chain $B)$ at the N-terminus. Data-collection and refinement statistics are summarized in Table 1 . The Ramachandran plot of the ferric ion-bound TtFbpA structure showed that 94 and $6 \%$ of the residues were in the most favoured and the additionally favoured regions, respectively. The ferric ion-bound TtFbpA protomer contains seven $\beta$-strands, $13 \alpha$-helices and one $3_{10}$-helix (Fig. $1 b$ ). The r.m.s.d. of the two ferric ion-bound TtFbpA protomers in the asymmetric unit was $0.8 \AA$ for 307 $\mathrm{C}^{\alpha}$ pairs.

\subsection{Anomalous difference Fourier map identifying the bound metal as iron}

In order to confirm that a ferric ion was bound to the putative metal-binding site of TtFbpA, a diffraction data set was collected from a crystal of ferric ion-bound TtFbpA at a wavelength of $1.7209 \AA$, the high-energy side of the absorption edge of iron. The crystal belonged to space group $P 2_{1} 2_{1} 2_{1}$, with unit-cell parameters $a=39.00, b=99.04, c=132.5 \AA$ (Table 1). The phases were calculated by the molecularreplacement method using the atomic coordinates of a TtFbpA protomer from the crystal structure of ferric ionbound TtFbpA as the search model and structural refinement was performed. In the resulting anomalous difference Fourier map a strong peak was observed at the predicted metalbinding site of each TtFbpA molecule, showing that iron was bound to the metal-binding site (Fig. 1c).

\subsection{The novel mode of coordination to the ferric ion by TtFbpA}

TtFbpA shows a novel type of coordination to a ferric ion (Table 2, Fig. $2 a$ ) in which the bound ferric ion is coordinated by three tyrosine residues in a manner similar to that observed in the binding in MhFbpA, a class 3 periplasmic ferric ionbinding protein. Despite this similarity, however, the manner of coordination differed between these two complexes (Figs. $2 a$ and $2 b$ ). The ferric ion bound to TtFbpA is six-coordinated by three tyrosine residues, two bicarbonates bound in a monodentate manner and a water molecule (Fig. 2a). The bound carbonic acid anions were identified as bicarbonates $\left(\mathrm{HCO}_{3}^{-}\right)$ rather than carbonates $\left(\mathrm{CO}_{3}^{2-}\right)$ because our ferric ion-bound TtFbpA crystal was obtained at $\mathrm{pH}$ 5.5, at which almost all carbonic acid anions are present as bicarbonate $\left(\left[\mathrm{HCO}_{3}^{-}\right]\right.$: $\left[\mathrm{CO}_{3}^{2-}\right]=63$ 000:1) based on the $\mathrm{p} K_{\mathrm{a}}$ values of 3.6 and 10.3 for $\mathrm{H}_{2} \mathrm{CO}_{3} \leftrightarrow \mathrm{HCO}_{3}^{-}+\mathrm{H}^{+} \leftrightarrow \mathrm{CO}_{3}^{2-}+2 \mathrm{H}^{+}$at $298 \mathrm{~K}$ and because both of the carbonic acid anions bind to the ferric ion in a monodentate manner rather than in a bidentate manner, suggesting that they are monovalent anions rather than divalent anions.

Very recently, another crystal structure of ferric ion-bound TtFbpA was reported (PDB entry 4elr; Wang et al., 2013) in which the bound ferric ion is five-coordinated by three tyrosine residues and a carbonate bound in a bidentate manner (Fig. 2c) as in MhFbpA (Shouldice et al., 2004). The different modes of coordination to the ferric ion observed in the two ferric ion-bound TtFbpA structures probably result from the different $\mathrm{pH}$ values used for crystallization: $\mathrm{pH} 5.5$ (this study) versus $\mathrm{pH} 7.5$ (Wang et al., 2013). Based on the abovementioned $\mathrm{p} K_{\mathrm{a}}$ values, bicarbonate is the predominant 
carbonic acid anion species at both $\mathrm{pH}$ values. However, the proportion of carbonate to bicarbonate is 100 -fold higher at $\mathrm{pH} 7.5\left(\left[\mathrm{HCO}_{3}^{-}\right]:\left[\mathrm{CO}_{3}^{2-}\right]=630: 1\right)$ than at $\mathrm{pH} 5.5\left(\left[\mathrm{HCO}_{3}^{-}\right]\right.$: $\left.\left[\mathrm{CO}_{3}^{2-}\right]=63000: 1\right)$ and a carbonate can bind to a ferric ion more tightly by bidentate binding than a bicarbonate. This explains why a carbonate rather than two bicarbonates binds to the ferric ion that is bound to TtFbpA at $\mathrm{pH} 7.5$ and also to MhFbpA at $\mathrm{pH} 7.5$ and Bordetella pertussis FbpA (BpFbpA) at $\mathrm{pH} 9.0$ (Fig. 2d).

The Gram-negative bacterium T. thermophilus HB8 can live in the wide $\mathrm{pH}$ range of 3.4-9.6 (Oshima \& Imahori, 1974; MicrobeWiki; http://microbewiki.kenyon.edu/index.php/ Thermus_thermophilus). We propose that $\mathrm{TtFbpA}$, a periplasmic protein from $T$. thermophilus $\mathrm{HB} 8$, can act as a ferric ion-binding protein over a wide $\mathrm{pH}$ range by adopting at least two different modes of coordination to a ferric ion depending on the $\mathrm{pH}$. This is the first example of a periplasmic ferric ironbinding protein that can coordinate a ferric ion via multiple types of coordination-complex formation.

\subsection{Comparison between the apo form and the ferric ion-bound form of TtFbpA}

Structural comparison of all of the crystal structures of TtFbpA has revealed that all of the apo TtFbpA structures at pH 5.2 (Fig. 3a; PDB entry 3wae; this study) and $\mathrm{pH} 7.5$ (Fig. 3c; PDB entries 4elo, 4elp and 4elq; Wang et al., 2013) and the ferric ion-bound TtFbpA structure at $\mathrm{pH} 5.5$ (Fig. 3b; PDB entry 3 waf; this study) adopt the open conformation, whereas the ferric ion-bound TtFbpA structure crystallized at $\mathrm{pH} 7.5$ (Fig. 3d; PDB entry 4elq; Wang et al., 2013) only adopts the closed conformation. The r.m.s.d. values for $\mathrm{C}^{\alpha}$ atoms between two open conformations are $0.3-0.8 \AA$, while those between the closed and open conformations are 2.2-2.6 $\AA$ (Table 3).

The crystal structures of apo TtFbpA and $\mathrm{Fe}^{3+}$-bicarbonates-TtFbpA at pH 5.2 and 5.5, respectively, show no significant structural differences, with r.m.s.d. values of $0.9 \AA$ for 307 $\mathrm{C}^{\alpha}$ pairs for chain $A$ and $1.1 \AA$ for $308 \mathrm{C}^{\alpha}$ pairs for chain $B$. To examine the molecular size of TtFbpA in solution in the absence and in the presence of ferric ions, gel-filtration

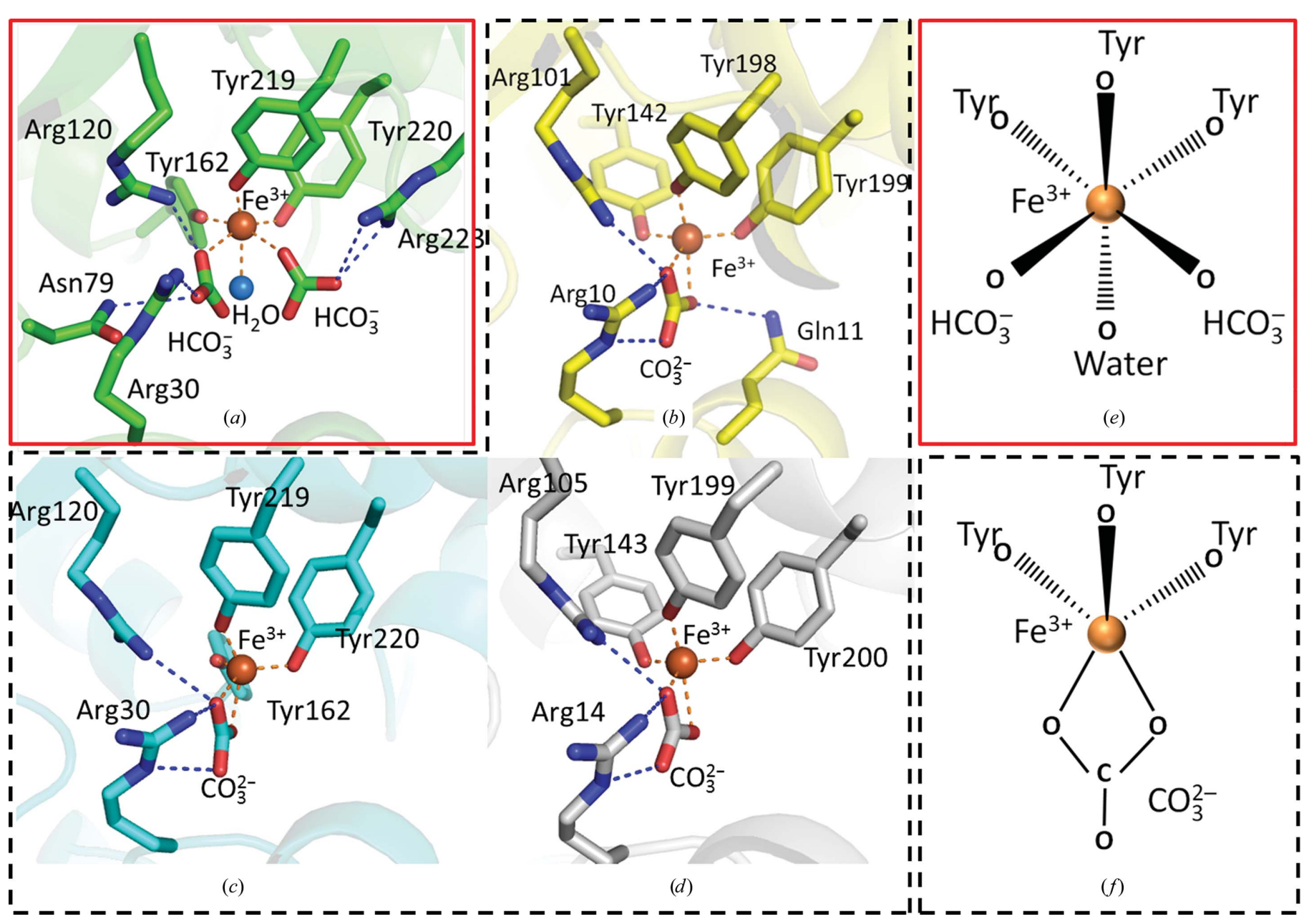

Figure 2

Comparison of the manner of coordination-complex formation with a ferric ion in TtFbpA, MhFbpA and BpFbpA. The metal-binding sites of $(a)$ Fe ${ }^{3+}-$ bicarbonates-TtFbpA (PDB entry 3waf; this work), (b) $\mathrm{Fe}^{3+}$-carbonate-MhFbpA (PDB entry 1si0; Shouldice et al., 2004), (c) Fe ${ }^{3+}-$ carbonate-TtFbpA (PDB entry 4elr; Wang et al., 2013) and $(d) \mathrm{Fe}^{3+}$-carbonate-BpFbpA (PDB entry 2owt; S. A. L. Tom-Yew, B. H. Shilton, E. G. Bekker, E. I. Tocheva \& M. E. P. Murphy, unpublished work) are shown. The three coordinating tyrosine residues are shown as stick models and the coordinated ferric ions and water molecules are shown as spheres. The orange and blue dotted lines represent coordinate and hydrogen bonds, respectively. The coordination in the six-coordinate $(e)$ and five-coordinate $(f)$ geometries of ferric ion is shown. 


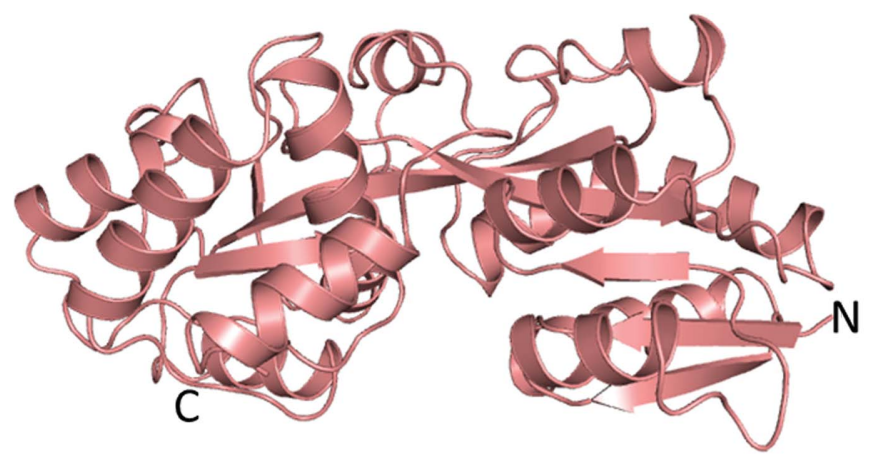

(a)

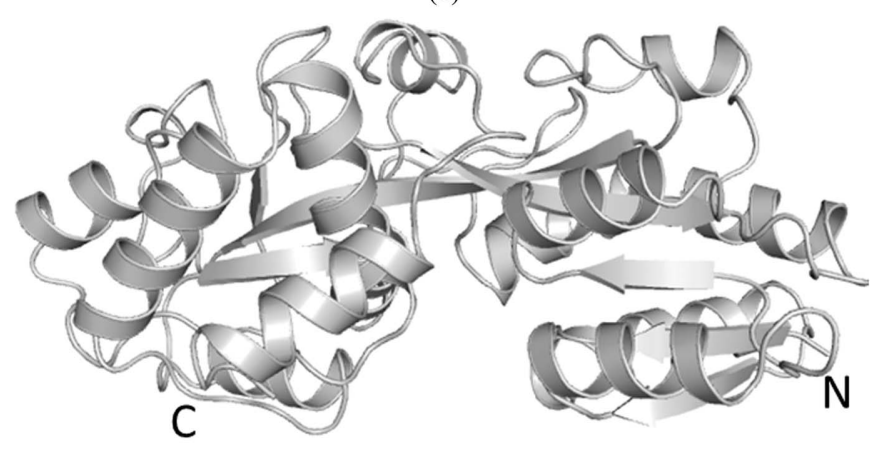

(c)

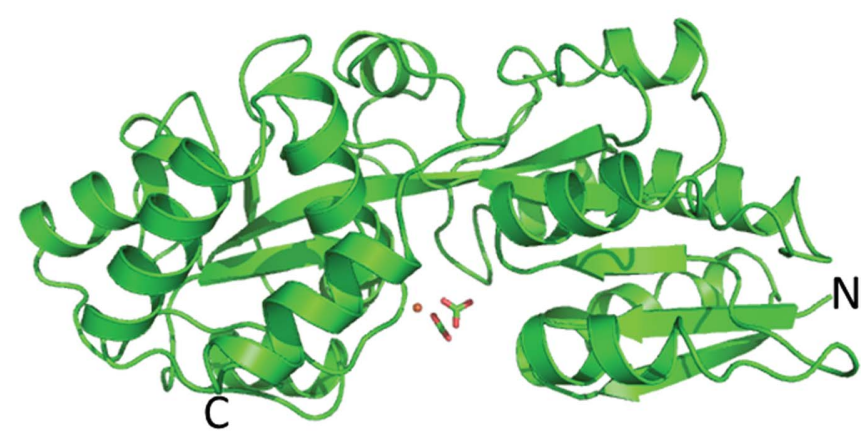

(b)

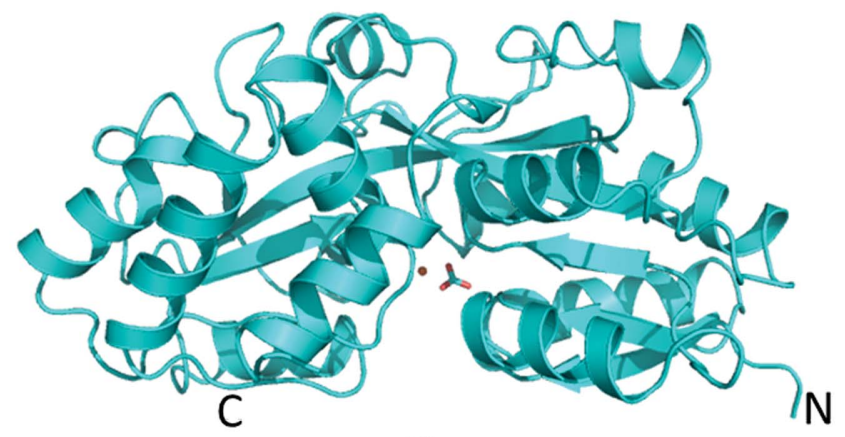

$(d)$

Figure 3

Structural comparison of apo TtFbpA and $\mathrm{Fe}^{3+}$-bound TtFbpA. (a) Apo TtFbpA (pH 5.2; PDB entry 3wae), (b) $\mathrm{Fe}^{3+}$-bicarbonates-TtFbpA (pH 5.5; PDB entry 3waf), (c) apo TtFbpA (pH 7.5; PDB entry 4elo) and (d) $\mathrm{Fe}^{3+}$-carbonate-TtFbpA (pH 7.5; PDB entry 4elr). (a), (b) and (c) are in the open conformation, whereas $(d)$ is in the closed conformation. The structures are aligned at their C-terminal domains.

Table 3

R.m.s.d.s $(\AA)$ of $\mathrm{C}^{\alpha}$ atoms between protomers of TtFbpA.

\begin{tabular}{|c|c|c|c|c|}
\hline & \multicolumn{2}{|c|}{$\begin{array}{l}\text { Apo TtFbpA (pH 5.2), } \\
\text { PDB code 3wae }\end{array}$} & \multicolumn{2}{|c|}{$\begin{array}{l}\mathrm{Fe}^{3+} \text {-bicarbonates- } \\
\text { TtFbpA (pH 5.5), } \\
\text { PDB entry 3waf }\end{array}$} \\
\hline & Chain $A$ & Chain $B$ & Chain $A$ & Chain $B$ \\
\hline \multicolumn{5}{|c|}{ Apo TtFbpA (pH 7.5), PDB code 4elo } \\
\hline Chain $A$ & 0.64 & 0.61 & 0.60 & 0.47 \\
\hline Chain $B$ & 0.58 & 0.49 & 0.48 & 0.43 \\
\hline Chain $C$ & 0.48 & 0.47 & 0.41 & 0.50 \\
\hline Chain $D$ & 0.66 & 0.44 & 0.56 & 0.41 \\
\hline Chain $E$ & 0.58 & 0.42 & 0.55 & 0.43 \\
\hline Chain $F$ & 0.70 & 0.50 & 0.56 & 0.45 \\
\hline \multicolumn{5}{|c|}{ Carbonate-TtFbpA (pH 7.5), PDB code 4elp } \\
\hline Chain $A$ & 0.53 & 0.40 & 0.52 & 0.52 \\
\hline Chain $B$ & 0.81 & 0.62 & 0.61 & 0.60 \\
\hline \multicolumn{5}{|c|}{ Carbonate-TtFbpA (pH 7.5), PDB code 4elq } \\
\hline Chain $A$ & 0.73 & 0.76 & 0.58 & 0.58 \\
\hline Chain $B$ & 0.75 & 0.56 & 0.64 & 0.45 \\
\hline Chain $C$ & 0.56 & 0.48 & 0.44 & 0.47 \\
\hline Chain $D$ & 0.69 & 0.49 & 0.58 & 0.41 \\
\hline \multicolumn{5}{|c|}{$\mathrm{Fe}^{3+}$-carbonate-TtFbpA ( $\mathrm{pH}$ 7.5), PDB code 4elr } \\
\hline Chain $A$ & 2.27 & 2.21 & 2.45 & 2.33 \\
\hline Chain $B$ & 2.26 & 2.21 & 2.45 & 2.33 \\
\hline
\end{tabular}

chromatography (GFC) and dynamic light-scattering (DLS) analyses were performed (data not shown). The GFCs at $\mathrm{pH}$ 5.0 and 8.0 indicated that both apo TtFbpA and ferric ionbound TtFbpA existed as monomers in solution and that ferric ion-bound TtFbpA eluted more slowly as a narrower peak than apo TtFbpA at both $\mathrm{pH}$ values, which suggests that the binding of ferric ion to TtFbpA should stabilize the closed conformation at both $\mathrm{pH}$ values. The hydrodynamic radii of apo TtFbpA and of ferric ion-bound TtFbpA obtained from the DLS data at pH 8.0 were $36 \pm 2$ and $29 \pm 1 \AA$, respectively. Since the longest diameters of apo TtFbpA and ferric ionbound TtFbpA in the crystal structures are approximately $60 \AA$, these GFC and DLS results show that TtFbpA exists as a monomer in solution regardless of the ferric ion binding and that $\mathrm{TtFbpA}$ adopts a more compact conformation in the ferric ion-bound state than in the apo state in solution, which is consistent with the proposed model of the ferric ionbinding/release cycle of FbpA (Shouldice et al., 2004) but is inconsistent with our crystal structures of apo TtFbpA and $\mathrm{Fe}^{3+}$-bicarbonates-TtFbpA both adopting the open conformation. This inconsistency may be owing to crystal-packing effects. Both structures were obtained from $P 2_{1} 2_{1} 2_{1}$ crystals with similar unit-cell parameters, which presumably favoured the open conformation rather than the closed conformation.

The synchrotron-radiation experiments were performed on beamlines NW12A and NE3A at the Photon Factory Advanced Ring, Ibaraki, Japan with the approval of the Photon Factory, KEK (Proposal No. 2011G190) and on beamline BL32XU at SPring-8, Hyogo, Japan with the approval of the Targeted Proteins Research Program Office at RIKEN/SPring-8 (Proposal No. 2011A1915). This work was partly supported by the Targeted Proteins Research Project (TPRP) and the Platform for Drug Discovery, Informatics and Structural Life Science of the Ministry of Education, Culture, Sports, Science and Technology (MEXT) of Japan and by 
Grants-in-Aid for Young Scientists (A) (17681026) and for JSPS Research Fellows (23-2637) from the Japan Society for the Promotion of Science (JSPS).

\section{References}

Braun, V. \& Killmann, H. (1999). Trends Biochem. Sci. 24, 104-109. Bruns, C. M., Anderson, D. S., Vaughan, K. G., Williams, P. A., Nowalk, A. J., McRee, D. E. \& Mietzner, T. A. (2001). Biochemistry, 40, 15631-15637.

Bruns, C. M., Nowalk, A. J., Arvai, A. S., McTigue, M. A., Vaughan, K. G., Mietzner, T. A. \& McRee, D. E. (1997). Nature Struct. Biol. 4, 919-924.

DeLano, W. L. (2002). CCP4 Newsl. Protein Crystallogr. 40, contribution 11.

Emsley, P. \& Cowtan, K. (2004). Acta Cryst. D60, 2126-2132.

Gerber, S., Comellas-Bigler, M., Goetz, B. A. \& Locher, K. P. (2008). Science, 321, 246-250.

Hollenstein, K., Frei, D. C. \& Locher, K. P. (2007). Nature (London), 446, 213-216.

Kabsch, W. (2010). Acta Cryst. D66, 125-132.

Kim, K., Okanishi, H., Masui, R., Harada, A., Ueyama, N. \& Kuramitsu, S. (2012). Proteomics, 12, 3063-3068.
Laskowski, R. A., MacArthur, M. W., Moss, D. S. \& Thornton, J. M. (1993). J. Appl. Cryst. 26, 283-291.

McCoy, A. J., Grosse-Kunstleve, R. W., Adams, P. D., Winn, M. D., Storoni, L. C. \& Read, R. J. (2007). J. Appl. Cryst. 40, 658-674.

Morris, R. J., Perrakis, A. \& Lamzin, V. S. (2003). Methods Enzymol. 374, 229-244.

Murshudov, G. N., Skubák, P., Lebedev, A. A., Pannu, N. S., Steiner, R. A., Nicholls, R. A., Winn, M. D., Long, F. \& Vagin, A. A. (2011). Acta Cryst. D67, 355-367.

Oshima, T. \& Imahori, K. (1974). Int. J. Syst. Evol. Microbiol. 24, $102-112$.

Shouldice, S. R., Dougan, D. R., Williams, P. A., Skene, R. J., Snell, G., Scheibe, D., Kirby, S., Hosfield, D. J., McRee, D. E., Schryvers, A. B. \& Tari, L. W. (2003). J. Biol. Chem. 278, 41093-41098.

Shouldice, S. R., McRee, D. E., Dougan, D. R., Tari, L. W. \& Schryvers, A. B. (2005). J. Biol. Chem. 280, 5820-5827.

Shouldice, S. R., Skene, R. J., Dougan, D. R., Snell, G., McRee, D. E., Schryvers, A. B. \& Tari, L. W. (2004). J. Bacteriol. 186, 3903-3910.

Tom-Yew, S. A. L., Cui, D. T., Bekker, E. G. \& Murphy, M. E. P. (2005). J. Biol. Chem. 280, 9283-9290.

Wang, Q., Chang, L., Wang, X. \& Liu, X. (2011). Acta Cryst. F67, 723-726.

Wang, Q., Lu, Q., Zhou, Q., Wang, X. \& Liu, X. (2013). Biochem. Biophys. Res. Commun. 434, 48-53. 